\title{
Avaliação com Doppler colorido e espectral da veia porta de cães
}

\author{
Evaluation of portal vein in dogs by color and spectral Doppler imaging
}

\author{
Raquel Sartor $^{\mathrm{I}^{*}}$ Maria Jaqueline Mamprim $^{\mathrm{I}}$
}

\section{- REVISÃO BIBLIOGRÁFICA -}

\section{RESUMO}

A perfusão total do fígado e suas contribuições relativas, pelo sistema portal e pela artéria hepática, podem estar alteradas por vários processos hepáticos, como doença hepato-celular difusa, neoplasia e shunts intra-hepáticos. $O$ estudo do comportamento do fluxo sangüíneo nos vasos do fígado por meio da ultra-sonografia Doppler tem demonstrado que este é um método viável não-invasivo e de grande auxílio, principalmente na avaliação da hemodinâmica portal. São duas as modalidades de ultra-sonografia Doppler com maior aplicação na rotina para avaliação de fluxo nos vasos abdominais, o Doppler espectral e o Doppler colorido. $\mathrm{Na}$ doença hepática crônica, ocorre alteração da complacência hepática devido à fibrose ou à cirrose, as quais acarretam alterações vasculares, levando ao aumento da pressão venosa portal. As principais indicações desse exame são os casos em que há suspeita de hipertensão portal. A hemodinâmica portal é avaliada pela mensuração de sua área, da velocidade média, do volume de fluxo no vaso e do índice de congestão portal. Esta revisão de literatura tem como objetivo descrever os princípios físicos básicos da ultra-sonografia Doppler e sua aplicação na avaliação da hemodinâmica portal nos cães.

Palavras-chave: ultra-sonografia, Doppler, veia porta, cães.

\section{ABSTRACT}

Total hepatic perfusion by portal system and hepatic arteria as well as its respective average contributions, can be affected for several hepatic processes, such as diffuse liver disease, neoplasm and intrahepatic shunts. Blood flow exams of the liver vessels using Doppler ultrasonography, appeared as a viable, non-invasive and helpful diagnostic method for the hemoportal dynamic evaluation. There are two
Doppler ultrasonographic modalities most frequently used in routine for evaluation of the abdominal vessels flow, spectral Doppler imaging and color-flow Doppler imaging. In chronic liver disease, alteration of hepatic complacency occurs because of either fibrosis or cirrhosis, bringing to vascular alterations which provoke an increase in portal pressure. The main indications for this exam are cases suspicious of portal hypertension. Portal hemodynamics is evaluated by measurements of the area, average speed, flow volume and portal vein congestion index. The objectives of this review are to describe the basic physical principles of Doppler ultrasonography and its use for evaluation of portal hemodynamics in dogs.

Key words: ultrasonography, Doppler, portal vein, dogs.

\section{INTRODUÇÃO}

O exame ultra-sonográfico é um método nãoinvasivo de avaliação dos órgãos e fornece muitas informações acerca das doenças hepáticas. Nos cães, os diagnósticos de lesões focais e difusas, doenças dos ductos biliares e alterações nas dimensões do fígado são amplamente descritos. A ultra-sonografia tem sido requisitada com freqüência para guiar biópsias e citologias aspirativas em órgãos abdominais, como o fígado. Embora essa série de manobras auxilie no diagnóstico das alterações hepáticas dos cães, ainda existe uma falha em pesquisar, ao contrário da medicina humana, a avaliação hepática baseada na

'Programa de Pós-graduação em Medicina Veterinária, Departamento de Reprodução Animal e Radiologia Veterinária, Universidade Estadual Paulista “Julio de Mesquita Filho". Distrito de Rubião Júnior, s/n, 18618-000, Botucatu, SP, Brasil. E-mail: raquelsartor@yahoo.com.br. *Autor para correspondência. 
hemodinâmica de seus vasos, sendo essa técnica pouco descrita na medicina veterinária (XIN \& CARLISIE, 1995).

A perfusão total hepática e suas contribuições relativas, pelo sistema portal e pela artéria hepática, podem estar alteradas por vários processos hepáticos, como doença hepato-celular difusa, neoplasia e shunts intra-hepáticos. O estudo do comportamento do fluxo sangüíneo nos vasos do fígado por meio da ultra-sonografia Doppler tem demonstrado que este é um método viável, nãoinvasivo, e de grande auxílio, principalmente na avaliação da hemodinâmica portal, pois, nos distúrbios vasculares hepáticos, as alterações clinicopatológicas e radiográficas podem não ser específicas e a ultrasonografia em modo bidimensional apenas informa acerca da arquitetura vascular, não caracterizando as condições do fluxo sangüíneo no vaso (KANTROWITZ et al., 1989).

Outros métodos disponíveis atualmente para avaliação da hemodinâmica vascular são a angiografia, a qual necessita de anestesia geral, e a cintilografia, que requer material específico de alto custo e difícil aquisição. Assim, a ultra-sonografia Doppler, que tem custo relativo baixo e pode ser realizada apenas com a contenção física do animal, tem sido um método muito utilizado na avaliação vascular hepática (D' ANJOU, 2007; KAMIKAWA, 2008).

Em humanos, o uso associado da modalidade Doppler em processos patológicos hepáticos revela resultados promissores. Os estudos visam principalmente estabelecer possíveis interações entre as alterações hemodinâmicas portais e as doenças hepáticas crônicas e também buscam caracterizar, segundo a vascularização detectada, diferentes tipos de neoplasias (NYLAND \& FISHER, 1990).

Na medicina veterinária, o efeito Doppler é utilizado desde o final da década de oitenta e desde então algumas pesquisas foram realizadas na tentativa de estabelecer padrões para o fluxo sangüíneo normal na veia porta de cães e suas possíveis alterações durante os processos hepáticos crônicos (NYLAND \& FISHER, 1990).

O objetivo desta revisão é descrever os princípios físicos da ultra-sonografia Doppler e sua aplicação na avaliação da hemodinâmica portal dos cães.

\section{Anatomia e fisiologia}

O fígado é a maior glândula do corpo e desempenha papel homeostático fundamental no equilíbrio de numerosos processos biológicos. Estimase que este órgão realize pelo menos 1500 funções bioquímicas essenciais para a sobrevida do animal. O fígado executa papel importante no metabolismo dos carboidratos, dos lipídios, das proteínas, das vitaminas e dos hormônios. Desempenha também funções de armazenamento e de detoxificação, além de funções imunológicas, hematológicas, secretórias e excretórias (SISSON, 1986; WEBSTER, 2005).

Na maioria dos cães, o fígado divide-se em seis lobos: quadrado, medial direito, medial esquerdo, lateral direito, lateral esquerdo e lobo caudado, este composto pelos processos caudado e papilar. A vesícula biliar situa-se entre os lobos quadrado e medial direito. A porta hepatis representa a fissura transversal na superfície ventral do fígado, onde a veia porta, a artéria hepática, o ducto biliar principal e os vasos linfáticos ingressam e saem do fígado (WEBSTER, 2005).

A irrigação hepática é feita por um sistema duplo, composto pelas artérias hepáticas e pelo sistema portal. Nos cães, cerca de $70 \%$ do sangue que chega ao fígado é transportado pela veia porta. Esta entra no fígado pelo hilo hepático, carregando sangue venoso oriundo dos intestinos, do pâncreas e do baço. Este sangue é rico em nutrientes, sendo essencial para as funções metabólicas realizadas no fígado, enquanto o sangue arterializado trazido pelos ramos da artéria hepática é responsável apenas pela nutrição do parênquima hepático (NICKEL, 1979).

A contribuição relativa do sistema portal e das artérias hepáticas para a irrigação do fígado varia com o estado fisiológico e também com as condições patológicas do organismo, como doença hepato-celular difusa, neoplasias e shunts intra-hepáticos. A veia porta, por ser principal responsável pela irrigação hepática, é também o vaso que mais sofre alterações hemodinâmicas quando doenças estão instaladas neste órgão, sendo assim, é o vaso de maior importância durante a avaliação com ultra-sonografia Doppler do fígado (KANTROWITZ et al., 1989; NYLAND \& FISHER, 1990).

O sistema portal é formado pela veia porta e seus afluentes. Este se inicia nos capilares viscerais e termina no fígado. A veia porta é formada pela confluência das veias mesentéricas cranial e caudal e da veia esplênica (veia lienal). O sistema portal transporta o sangue venoso do pâncreas, do baço e de todo trato gastrintestinal, exceto do canal anal (KANTROWITZ et al., 1989; EVANS, 1993). Este sangue é um dos fatores que determinam as dimensões hepáticas, pois é rico em fatores hepatotróficos, os quais determinam as dimensões de cada hepatócito (WEBSTER, 2005).

A familiaridade com a anatomia normal do sistema hepatobiliar melhora as condições do clínico 
para o uso e a interpretação dos exames laboratoriais e do ultra-som, para a consideração dos diagnósticos diferenciais apropriados, e para a seleção dos tratamentos diante de um diagnóstico histopatológico (WEBSTER, 2005).

\section{Ultra-sonografia}

Por meio da ultra-sonografia, pode-se analisar a anatomia intra-hepática de maneira segura e não-invasiva e acrescentar informações ao diagnóstico, mesmo antes dela ser observada pela radiografia convencional ou pelos exames laboratoriais. As indicações para exame ultra-sonográfico hepático incluem hepatomegalia, massas abdominais, icterícia, ascite, suspeita de ruptura diafragmática, perda de peso, pesquisas de metástases hepáticas, guia de biópsias e monitoração de tratamentos (MAMPRIM, 2004).

O conhecimento da anatomia venosa ajuda a identificar regiões específicas do fígado durante o exame ultra-sonográfico (NYLAND \& MATTOON, 2004). Para identificação dos diversos ramos da veia porta, estes devem ser diferenciados das veias hepáticas. Essa distinção é feita pela localização anatômica dos ramos principais, pela sua distribuição e pela ecogenicidade da parede dos vasos (XIN \& CARLISLE, 1995).

Os ramos portais apresentam parede ecogênica, enquanto as veias hepáticas normalmente não têm as paredes visíveis, assim, estas são visibilizadas apenas quando a onda de ultra-som e o vaso estão perpendiculares (SZATMARI et al., 2001).

Para a realização do exame ultra-sonográfico dos ramos portais, o paciente deve ser posicionado em decúbito dorsal ou lateral esquerdo. A veia porta é visibilizada tanto pelo acesso ventral como pelo intercostal direito, entre o $8^{\circ}$ e o $11^{\circ}$ espaço intercostal, podendo, através destes, ser observada entrando no fígado, no hilo hepático (EVANS, 1993; NYLAND \& MATTOON, 2005).

Para que seja possível determinar parâmetros ao Doppler, como o volume de fluxo sangüíneo no vaso, é importante realizar a mensuração dos diâmetros e das áreas da veia porta, da artéria aorta e da veia cava caudal, pois doenças hepáticas, assim como alterações cardíacas, podem alterar esses valores. Para essa manobra, é feito corte transversal dos vasos, com o paciente posicionado em decúbito lateral esquerdo, sendo o corte realizado na altura do $11^{\circ}$ espaço intercostal direito (NYLAND \& MATTOON, 2005). Sabe-se que vários fatores podem alterar essa área, como as variações respiratórias e posturais e o estado pós-prandial. Dessa forma, as mensurações devem ser sempre realizadas com os indivíduos em jejum, no mesmo decúbito e com a respiração tranqüila (MACHADO et al., 2004).

A área da veia porta de cães sadios foi mensurada em dois trabalhos, os quais encontraram média de $0,66 \pm 0,14 \mathrm{~cm}^{2}$, na avaliação de 16 cães da raça Beagle, pesando entre 11 e 19,5kg (LAMB \& MAHONEY, 1994), e de $0,65 \pm 0,15 \mathrm{~cm}^{2}$, quando avaliados 10 cães, sem raça definida, que pesavam entre 16 e 26kg(NYLAND \& FISHER, 1990)

Ultra-sonografia Doppler

Princípios físicos

O efeito Doppler é uma característica observada nas ondas, quando emitidas ou refletidas, por um objeto que está em movimento, em relação a um observador. Esse nome é utilizado em homenagem ao físico austríaco Johann Christian Andréas Doppler, que o descreveu pela primeira vez em 1842 (CERRI et al., 1998).

No exame de ultra-som em modo-B, o transdutor e o tecido observado estão parados, assim a freqüência da onda emitida pelo transdutor é a mesma da refletida pelos tecidos. Na ultra-sonografia Doppler dos vasos sangüíneos, o objeto parado é o transdutor e os refletores são as hemácias, as quais estão em movimento e, dessa maneira, geram um eco de retorno com freqüência diferente da emitida pelo transdutor. A diferença entre a freqüência do som emitido e a do som refletido é chamada de desvio de freqüência Doppler ou desvio Doppler (CERRI et al., 1998; SZATMARI et al., 2001; GINTHER \& UTT, 2004).

Se o fluxo sangüíneo mover-se em direção ao transdutor, então a variação Doppler será positiva, ou seja, o eco de retorno terá maior freqüência. Se o fluxo estiver se afastando do transdutor, então a variação Doppler será negativa e a freqüência do eco de retorno será menor do que a do emitido (CERRI et al., 1998; SZATMARI et al., 2001; GINTHER \& UTT, 2004; NYLAND \& MATTOON, 2005).

A variação na freqüência é audível ao ouvido humano, ou seja, abaixo de $20 \mathrm{kHz}$, diferente da onda emitida pelo transdutor, que está geralmente entre 2 a $10 \mathrm{MHz}$. Esse som audível, quando interpretado por um operador experiente, pode ser utilizado como um método instantâneo para serem obtidas informações qualitativas adicionais à avaliação do gráfico no Doppler espectral (GINTHER \& UTT, 2004).

Quanto maior a variação Doppler, maior será a velocidade que a hemácia está se deslocando. Entretanto, essa variação só será máxima, ou seja, o efeito Doppler ideal será obtido quando o vaso sangüíneo estiver paralelo ao feixe de ultra-som transmitido (NYLAND \& MATTOON, 2005). A equação 
Doppler descreve essa relação:

$\Delta \mathrm{f}=2 \mathrm{f} v \cos \alpha / \mathrm{c}$ na qual $\Delta \mathrm{f}$ é a freqüência de variação Doppler (diferença entre a freqüência do som emitido e do refletido), f é a freqüência original do transdutor, v é a velocidade de movimento das hemácias, a é o ângulo entre o feixe de ultra-som incidente e o eixo longitudinal do segmento estudado (do vaso sangüíneo) e c é a velocidade de propagação do som nos tecidos (1540m $\left.\mathrm{S}^{-1}\right)$.

Dessa maneira, para se obter um efeito Doppler máximo, o vaso deve estar paralelo ao feixe de ultra-som, obtendo um ângulo de incidência igual a zero, pois co-seno de zero é igual a um. Assim, a variação de freqüência máxima é produzida e o ângulo tem efeito mínimo nos resultados calculados. A obtenção do ângulo igual a zero é muito difícil na avaliação de vasos periféricos, assim, para um exame de ultra-sonografia Doppler realizar medidas com poucas chances de erros, autores recomendam que sejam utilizadas apenas medidas feitas com o ângulo de insonação de até $60^{\circ}$ (SZATMARI et al., 2001; BOOTE, 2003; ROSE \& NELSON, 2004).

\section{Doppler espectral}

Nos equipamentos ultra-sonográficos mais modernos, é possível associar a imagem do Doppler espectral à imagem em modo-B. Por unir as duas formas de imagem, a técnica é também conhecida como duplex Doppler. A composição dos dois métodos fornece diagnósticos anatômico e funcional concomitantes. A detecção dos sinais gerados pela movimentação dos glóbulos vermelhos ocorre no interior de uma pequena área, chamada de volume de amostra, a qual pode ser colocada em qualquer local do vaso. Obtém-se assim a curva da velocidade nos pontos em que se deseja analisar o fluxo sangüíneo (CERRI et al., 1998; NYLAND \& MATTOON, 2005).

A análise da curva de velocidade dada em função do tempo traz informações qualitativas como presença ou não de fluxo na região analisada, direção e sentido do fluxo e avaliação da morfologia das ondas espectrais. Fornece também informações semiquantitativas, pois, de acordo com a morfologia do espectro adquirido, pode-se supor a condição do fluxo no vaso analisado e informações quantitativas como medidas de velocidade, em especial máxima e mínima, a partir das quais podem ser calculados os índices de resistividade (IR) e de pulsatilidade (IP), que analisam a impedância vascular (CARVALHO, 2007). Nesse método, o mesmo cristal piezoelétrico que emite a onda sonora a capta. A emissão da onda é de curta duração (1\% do ciclo) e alterna-se com longos períodos de espera (99\% do ciclo) (GINTHER \& UTT, 2004).
O número de pulsos emitidos por segundo, denominado de freqüência de repetição de pulsos (PRF), determina o poder de alcance e a freqüência máxima detectada pelo transdutor. A PRF deve ser adequada às características de freqüências dos sinais captados, ou seja, quanto maior a variação de freqüência gerada pelo fluxo de alta velocidade, maior deve ser a PRF. Variações de freqüência maiores do que a metade do PRF serão representadas como se estivessem na direção contrária, devido à geração de um artefato do Doppler chamado de aliasing (CERRI et al., 1998; GINTHER \& UTT, 2004; NYLAND \& MATTOON, 2005).

\section{Doppler colorido}

O Doppler colorido é a mais recente tecnologia da ultra-sonografia diagnóstica. O método é uma fusão da escala de cinza e da detecção de movimentos, produzindo uma imagem que representa os tecidos moles em escala de cinza e fluxo sangüíneo em escala colorida. Sinais de células vermelhas em movimento são visibilizados em cores, em função de suas movimentações, no mesmo sentido ou em sentido contrário ao transdutor. A atribuição de cores (vermelho e azul) para cada sentido de fluxo é determinada pelo operador. Convencionou-se que o fluxo em direção ao transdutor é representado em vermelho e em direção contrária ao transdutor, em azul (CERRI et al., 1998; NYLAND \& MATTOON, 2005; CARVALHO, 2007).

A tonalidade da cor representa a velocidade das hemácias, quando se adiciona branco a uma cor significa que o fluxo naquela região é mais rápido, por exemplo, um vaso visibilizado em azul-escuro representa fluxo afastando-se do transdutor e um azulclaro também indica o mesmo sentido de fluxo, mas com maior velocidade (POWIS, 1994).

\section{Avaliação da hemodinâmica portal} Medicina

A utilização do Doppler na medicina foi importante para expandir a aplicação clínica da ultrasonografia e o desenvolvimento da técnica e dos equipamentos proporcionou um melhor entendimento da hemodinâmica vascular e da correlação entre as alterações no fluxo sangüíneo e os diversos processos patológicos (BOOTE, 2003). A avaliação da vascularização hepática tornou o exame ultrasonográfico mais completo, pois com a técnica passou a ser possível diferenciar estruturas vasculares e nãovasculares, como nos casos em que a ultra-sonografia em modo-B falha em distinguir um ducto biliar dilatado de um vaso portal. Essa técnica permitiu avaliar a velocidade do fluxo sangüíneo na veia porta, que, 
quando diminuída, é um importante indicativo da presença de hipertensão portal e tornou possível também determinar a direção do fluxo sangüíneo, detectando, assim, a presença de fluxos hepatofugais no sistema portal, a qual indica ao clínico a maior gravidade do quadro de uma doença hepática crônica (CERRI et al., 1998).

Dessa maneira, essa técnica representou um marco no exame de pacientes portadores de hipertensão portal, permitindo a avaliação da dinâmica vascular de forma não-invasiva. Atualmente, recomenda-se a inclusão do exame na avaliação inicial de pacientes hepatopatas, sendo este de extrema importância na conduta clínica/cirúrgica destes pacientes (MACHADO et al., 2004).

LEMOS (2002) descreveu que os primeiros relatos da utilização da ultra-sonografia Doppler na avaliação da hemodinâmica portal foram realizados por GILL (1979 e 1985), buscando mensurar o volume de fluxo na veia porta (VFVP). MORIYASU et al. (1984) afirmaram que o método era confiável para avaliação do VFVP e apenas um ano depois OHNISHI et al. (1985) publicaram dois estudos que verificaram a diminuição da velocidade de fluxo na veia porta em doentes cirróticos, porém, sem a observação de alterações no VFVP. MORIYASU et al. (1986) voltaram a publicar novos artigos reafirmando a aplicabilidade do Doppler no estudo da hemodinâmica portal. Além disso, os autores sugeriram novo parâmetro para pesquisa, chamado por eles de índice de congestão portal (ICP).

Atualmente, a avaliação pela ultrasonografia Doppler em pacientes com hepatopatias tem sido amplamente utilizada na medicina. Na doença hepática crônica, ocorre alteração da complacência hepática devido à fibrose ou à cirrose, as quais acarretam alterações vasculares, levando ao aumento da pressão venosa portal. As principais indicações desse exame são casos em que há suspeita de hipertensão portal, sendo este realizado como uma avaliação inicial, pois pode fornecer informações importantes à conduta clínica (O’DONOHUE et al., 2004).

Nesses pacientes a principal avaliação realizada com Doppler é a da veia porta. A hemodinâmica portal vinha sendo avaliada pela mensuração de sua área, da velocidade média do fluxo (Vm) e do volume de fluxo portal (VFVP) até 1986, sendo que após essa data a avaliação passou a ser feita também por meio do índice de congestão portal.

Em 1986, MORIYASU et al., na tentativa de introduzir o ICP como parâmetro na avaliação de pacientes com hipertensão portal, compararam os valores da área, da Vm, do VFVP e do ICP na veia porta em cinco grupos de pacientes humanos. $\mathrm{O}$ primeiro grupo era composto por voluntários sadios, o segundo por pacientes portadores de hepatite aguda, o terceiro por portadores de hepatite crônica ativa, o quarto por portadores de cirrose hepática e o quinto grupo por portadores de hipertensão portal idiopática.

Em seu estudo, MORIYASU et al. (1986) constataram um aumento significativo da área da veia porta nos doentes em relação ao grupo controle. $\mathrm{Na}$ comparação dessa variável entre os grupos, apenas os pacientes portadores de hepatite aguda não diferenciaram do grupo controle. A Vm também estava alterada nos enfermos, com exceção dos portadores de hepatite aguda, com diminuição significativa em seu valor em relação ao grupo controle. O VFVP não foi estatisticamente diferente entre nenhum dos grupos e o ICP foi maior nos pacientes doentes em relação ao grupo controle. Ao mesmo tempo foi aferida a pressão venosa portal através de um cateter e uma fraca correlação positiva foi observada entre o ICP e a pressão venosa portal (MORIYASU et al., 1986).

Em 2002, SUGIMOTO et al. demonstraram que tanto a Vm como o VFVP estavam significativamente diminuídos em pacientes com cirrose em relação ao grupo controle e a pacientes portadores de hepatite crônica, porém, estes últimos não foram diferentes quando comparados ao grupo de pacientes hígidos. Diante dos resultados deste estudo foi possível concluir que as medidas de Vm e de VFVP são parâmetros importantes na avaliação de pacientes com lesão hepática severa, mas não na avaliação de pacientes com lesão hepática de grau moderado.

Ainda em 2002, PISCAGLIA et al. avaliaram pacientes portadores de esplenomegalia causada por diferentes enfermidades, tais como hepatite crônica, cirrose, desordens hematológicas e pacientes que já haviam realizado transplante hepático. O estudo visava estabelecer parâmetros ao Doppler espectral que diferenciassem a causa primária desta esplenomegalia entre hipertensão portal cirrótica e distúrbios hemodinâmicos, uma vez que entre 52 e $83 \%$ dos pacientes com hipertensão portal apresentam aumento de volume do baço. O autor concluiu que, para diferenciar a causa da esplenomegalia como sendo de um distúrbio hematológico ou de uma hipertensão portal cirrótica, o melhor parâmetro avaliado ao Doppler na veia porta é a Vm.

$\mathrm{Na}$ hipertensão portal em grau mais avançado, também é possível detectar fluxo hepatofugal ao Doppler, o qual é considerado anormal em qualquer segmento da veia porta. A direção invertida do fluxo pode ser visibilizada ao Doppler colorido ou ao Doppler espectral, observando fluxo portal e fluxo arterial hepático em direções opostas (WACHSBERG et al., 2002).

Ciência Rural, v.39, n.2, mar-abr, 2009. 


\section{Medicina veterinária}

Na medicina veterinária, pesquisas com ultra-sonografia Doppler hepática foram inicialmente realizadas no final da década de 80. Desde então alguns estudos buscaram determinar valores de volume e velocidade de fluxo na veia porta, assim como avaliar as veias hepáticas e a artéria hepática em cães sadios e portadores de hepatopatias.

As avaliações da veia porta e seus ramos pelo Doppler colorido são de grande valor para determinar a existência e o sentido de seu fluxo (O’DONOHUE et al., 2004). O fluxo da veia porta nos cães é hepatopetal, ou seja, em direção ao fígado, ao passo que o fluxo hepatofugal, isto é, em direção oposta ao fígado, é considerado anormal em qualquer segmento deste vaso. Esse achado é clinicamente importante no diagnóstico da hipertensão portal para determinar a presença de shunts portossistêmicos e auxiliar no diagnóstico de pacientes com cirrose (WACHSBERG et al., 2002).

Na avaliação, procede-se determinando o segmento do vaso a ser analisado colocando o cursor do Doppler espectral no local previamente estabelecido com o auxílio do Doppler colorido. Nessa etapa, é importante escolher um segmento em que seja possível se obter o menor ângulo de insonação para que as medidas realizadas sejam menos susceptíveis a erros, sendo que a obtenção deste ângulo na veia porta principal do cão é difícil, havendo, muitas vezes, a necessidade de se trabalhar com ângulos acima do indicado (KANTROWITZ et al., 1989; NYLAND \& FISHER, 1990; LAMB \& MAHONEY, 1994; SUGIMOTO et al., 2002).

Em 2002, foi demonstrado que o acesso aos ramos intra-hepáticos da veia porta normalmente proporcionam um menor ângulo de insonação, obtendo resultados mais confiáveis, principalmente em relação às medidas de velocidades e aos demais índices que as utilizam em seus cálculos. Os autores também afirmam que o ramo direito da veia porta apresenta alterações como diminuição na velocidade média e no volume de fluxo sangüíneo, diretamente relacionadas aos danos causados pela cirrose (SUGIMOTO et al., 2002).

Após a correção angular, pode-se medir a velocidade média $(\mathrm{Vm})$ do fluxo no vaso, sendo esta medida por meio do gráfico formado pela velocidade em função do tempo. Para entender como a velocidade é calculada, é importante conhecer como se comporta o fluxo sangüíneo na veia porta.

O fluxo da veia porta é quase contínuo, com baixa pulsatilidade, sofrendo pequenas variações com o ciclo cardíaco e os movimentos respiratórios. A variação de velocidades dentro do vaso é grande, pois seu fluxo é laminar e classificado como parabólico, ou seja, é um fluxo sem turbulências, com velocidades maiores no centro do vaso e menores em sua periferia, próximo à parede (KANTROWITZ et al., 1989).

Existem três métodos propostos para obtenção do gráfico e do cálculo da velocidade, porém, são dois os mais utilizados: o método da insonação uniforme e o método da velocidade máxima. No método da insonação uniforme, utiliza-se como volume de amostra todo o vaso, ultrapassando levemente suas paredes. Assim, obtém-se diretamente a velocidade média do fluxo, pois, com um volume de amostra que abrange todo o diâmetro do vaso, são analisadas tanto as velocidades maiores contidas no centro como as velocidades menores próximas às paredes do vaso (NYLAND \& FISHER, 1990; LAMB \& MAHONEY, 1994). No método da velocidade máxima, posiciona-se a amostra no centro do vaso e esta deve abranger apenas a metade de seu diâmetro. Esse método utiliza como amostra apenas as hemácias que se movem com maior velocidade, ou seja, aquelas que estão no centro do vaso e assim é obtida a velocidade máxima do fluxo. A velocidade média ( $V m$ ) é calculada multiplicando este valor por 0,57 , uma vez que é considerada como sendo 57\% da velocidade máxima, como determinado em estudo in vitro e in vivo, em modelo circulatório experimental realizado por MORIYASU et al., (1986) a fim de determinar essa constante em humanos, a qual foi adaptada aos cães por NYLAND \& FISHER (1990), mostrando-se compatível para a espécie. Também foi observado que a velocidade varia durante a respiração, sendo maior durante a expiração e menor durante a inspiração (NYLAND \& FISHER, 1990; LAMB \& MAHONEY, 1994). Esta variação já foi atribuída a uma possível compressão da veia porta pelo diafragma durante a inspiração diminuindo a velocidade de seu fluxo, porém, atualmente é explicada pelo fato de a amostra do Doppler espectral mover-se no interior do vaso durante os movimentos respiratórios, captando alternadamente fluxos mais velozes no centro e mais lentos na periferia do vaso. Portanto, a utilização do método da insonação uniforme seria mais vantajosa, pois diminuiria esta variação, apesar de os autores não terem encontrado diferença estatisticamente significativa entre os dois métodos (LAMB \& MAHONEY, 1994).

O valor encontrado por LAMB \& MAHONEY (1994) para a Vm na veia porta foi de $14,7 \pm 2,5 \mathrm{~cm} \mathrm{~s}^{-1}$. Para o trabalho, foram utilizados 16 cães sadios da raça beagle, machos e fêmeas, pesando entre 11 e 19,5kg. NYLAND \& FISHER (1990) encontraram a Vm na veia porta igual a $18,1 \pm 7,6 \mathrm{~cm} \mathrm{~s}^{-1}$, utilizando 10 cães sadios, machos e fêmeas, sem raça definida, pesando de 16 a $26 \mathrm{~kg}$. 
A mensuração do VFVP tem importância clínica, uma vez que esse volume representa de 65 a $85 \%$ do volume sangüíneo total recebido pelo fígado, podendo variar com o estado fisiológico do paciente $\mathrm{e}$ também em condições patológicas, como citado anteriormente. Com a área e a velocidade calculadas, é possível calcular o VFVP (ml min-1 $\left.\mathrm{kg}^{-1}\right)$. Para o cálculo, foi proposta uma fórmula, a qual foi analisada em humanos e foi constatado um erro de apenas 10 a $15 \%$ (KANTROWITZ et al., 1989): VFVP = Vm xA/p na qual p representa o peso corpóreo medido em kg. Para o cálculo, deve-se ter atenção, pois é necessário utilizar a Vm em cm min ${ }^{-1}$.

NYLAND \& FISHER (1990) estabeleceram que a Vm é $57 \%$ da velocidade máxima na veia porta. Assim, a Vm foi obtida por duas maneiras diferentes para que o VFVP fosse calculado por meio da mensuração da velocidade máxima do fluxo multiplicando esse valor por 0,57 e pelo método da insonação uniforme, o qual fornece diretamente a Vm. Posteriormente, foi calculado o VFVP, cujo valor médio obtido foi de $31,2 \pm 9,8 \mathrm{ml} \mathrm{min}^{-1} \mathrm{~kg}^{-1}$. LAMB \& MAHONEY (1994) calcularam o VFVP em cães da raça

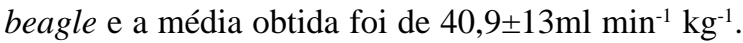
Outro cálculo que deve ser efetuado na avaliação da hemodinâmica portal é o índice de congestão portal (ICP). Este índice relaciona a área com a Vm do fluxo na veia porta, pela fórmula: $\mathrm{ICP}=\mathrm{A} / \mathrm{Vm}$.

MORIYASU et al.(1986) encontraram o valor de $0,070 \pm 0,029 \mathrm{~cm} \mathrm{~s}^{-1}$ para o ICP na veia porta de humanos sadios. Em cães, os valores obtidos foram de 0,046 $\pm 0,012$ (LAMB \& MAHONEY, 1994) e 0,041 $\pm 0,018 \mathrm{~cm} \mathrm{~s}^{-1}$ (NYLAND \& FISHER, 1990). Vários fatores podem alterar o ICP, tais como pressão venosa portal, resistência vascular intra-hepática, fluxo de sangue portal e formação de circulação colateral portossistêmica. O tempo de ocorrência do processo também é importante, pois as alterações patológicas na veia porta são progressivas (MORIYASU et al., 1986; FERREIRA et al., 2005).

Na medicina veterinária, apenas um trabalho foi realizado seguindo a mesma linha de pesquisa da medicina, buscando constatar possíveis alterações ao Doppler na veia porta de cães portadores de hipertensão portal. A pesquisa foi realizada em 1990 por NYLAND \& FISHER, buscando estabelecer valores de normalidade e possíveis variações no fluxo da veia porta de cães. O estudo comparou animais sadios e portadores de cirrose induzida experimentalmente, sendo que foram utilizados 10 cães, sem raça definida, machos e fêmeas, que pesavam entre 16 e $26 \mathrm{~kg}$. Os animais foram avaliados pela ultra-sonografia Doppler e posteriormente cirrose foi induzida por meio da ligadura do ducto biliar comum. A instalação do quadro foi confirmada pela histopatologia durante a necropsia dos animais. Após quatro semanas, os animais foram reexaminados e não foi verificada diferença na área da veia porta entre o grupo controle e os animais cirróticos. Diminuição significativa foi observada nos valores da Vm e do VFVP nos animais com cirrose em relação ao grupo controle, sendo observados os valores de $9,2 \pm 1,70 \mathrm{~cm} \mathrm{~s}^{-1}$ e de $17,2 \pm 4,9 \mathrm{ml} \mathrm{min} \mathrm{mg}^{-1}$, respectivamente. O ICP nos animais cirróticos estava aumentado em relação aos animais sadios, com valor médio de $0,062 \pm 0,018 \mathrm{~cm} \mathrm{~s}^{-1}$.

Em pacientes humanos, é relatado que o fluxo sangüíneo portal normal mantém-se até a fase mais tardia da doença, quando este se torna reverso. Porém, o ICP apresenta-se elevado nesses pacientes porque ocorre um aumento do calibre da veia porta enquanto a velocidade de seu fluxo diminui. Nos cães estudados por NYLAND \& FISHER (1990), não foi observada alteração no calibre do vaso, porém, o ICP mostrou-se elevado em razão da diminuição da velocidade do fluxo no vaso, permitindo um diagnóstico precoce da alteração hepática induzida experimentalmente.

\section{CONSIDERAÇÕES FINAIS}

A ultra-sonografia Doppler é muito utilizada na medicina para a avaliação de hepatopatas, representando um marco no diagnóstico de portadores de hipertensão portal, sendo atualmente um exame indispensável na avaliação destes pacientes. No Brasil, a ultra-sonografia Doppler dos principais vasos que constituem o sistema de irrigação e drenagem hepática nos cães foi introduzida recentemente na rotina clínica. A técnica oferece informações adicionais àquelas obtidas com o ultra-som convencional e permite a associação da imagem da anatomia em tempo real às informações dinâmicas do fluxo sangüíneo.

Com a utilização do exame Doppler no fígado, é possível verificar a presença, a direção e o tipo de fluxo em seus vasos sangüíneos, mensurar velocidades e volume de fluxo, além de índices correlacionados à impedância vascular. Tais dados refletem a complacência do parênquima hepático, indicando, assim, a presença ou não de afecções neste órgão.

Pesquisas acerca da avaliação da veia porta de cães sadios e portadores de doença hepática crônica sugeriram que a técnica proporciona informações que auxiliam o clínico no diagnóstico, na conduta e no prognóstico das hepatopatias, particularmente nos casos de cirrose hepática. 
Os experimentos até agora desenvolvidos por NYLAND \& FISHER (1990) e por LAMB \& MAHONEY (1994), que padronizaram os valores de velocidade, do volume de fluxo e do índice de congestão da veia porta de cães, restringem-se a duas faixas de peso, de 16,7 a $26,7 \mathrm{~kg}$ e de 11,2 a $19,5 \mathrm{~kg}$, respectivamente, além de terem sido realizados com um pequeno número amostral. Na experiência dos autores desta revisão, essas condições limitam a utilização dessa técnica na rotina, uma vez que algumas das variáveis obtidas na veia porta utilizam as áreas dos vasos em seus cálculos e estas variam de acordo com o porte do animal. A constatação de resultados que não são compatíveis com os valores determinados traz a incerteza da real presença de um processo patológico, particularmente nos cães com portes distintos dos relatados. Essa premissa pode ser justificada pelo índice de congestão, o qual relaciona a área com a velocidade da veia porta. Dessa maneira, esse índice seria o mais afetado, se fosse tomado como padrão apenas o resultado dos ensaios citados anteriormente, pois não seria considerado o fato que animais de portes diferentes têm valores de áreas distintos e tal variação poderia ser a causa da divergência entre o valor obtido e aquele considerado normal.

Assim, para que seja possível empregar a ultra-sonografia Doppler com maior eficiência e clareza na avaliação de cães portadores de hepatopatias, é importante o conhecimento das possíveis variações que ocorrem dentro da normalidade nos diferentes portes de cães atendidos na rotina clínica.

\section{REFERÊNCIAS}

BOOTE, E.F. AAPM/RSNA Physics tutorial for residents: topics in us. Radiographics, Chicago, v.23, p.1315-1327, 2003.

CARVALHO, C.F. Princípios básicos do doppler em ultrasonografia. In: CARVALHO, F.C. Curso de fundamentos e aplicações do ultra-som doppler em veterinária. São Paulo: PROVET, 2007.

CERRI, G.G. et al. Doppler. São Paulo: Sarvier, 1998. 271p.

D’ ANJOU, M. A. The sonographic search for portosystemic shunts. Clin Tech Small Anim Pract, v.22, p.104-114, 2007.

EVANS, H.E. Miller's anatomy of the dog. 3.ed. Philadelphia : Saunders, 1993. 1113p.

FERREIRA, F.G. et al. Índice de congestão portal e a ocorrência de trombose portal pós-dape. Rev Assoc Med Bras, São Paulo, v.51, n.4, p.1-9, 2005.

GILL, R.W. Pulsed Doppler with B-mode imaging for quantitative blood flow measurement. Ultrasound in Med and Biol, Chicago, v.5, p.223-235, 1979.
GILL, R.W. Measurement of blood flow by ultrasound accuracy and sources of error. Ultrasound in Med and Biol, Chicago, v.2, p. 625-641, 1985.

GINTHER, O.J.; UTT, M.D. Doppler ultrasound in equine reproduction: principles, techniques, and potential. J Equine Vet Sci, Madison, v.24, n.12, p.516-526, 2004.

KAMIKAWA, L. Avaliação morfométrica e hemodinâmica comparativa dos vasos envolvidos no desvio portossistêmico em cães. 2008. 96f. Tese (Doutorado, área de anatomia dos animais domésticos e silvestres) Universidade de São Paulo, São Paulo.

KANTROWITZ, B.M. et al. Estimation of portal blood flow using duplex real-time and pulsed Doppler ultrasound imaging in the dog. Vet Radiol, Malden, v.30, n.5, p.222-226, 1989.

LAMB, C.R.; MAHONEY, P.N. Comparision of three methods for calculating portal blood flow velocity in dogs using duplexDoppler ultrasonography. Vet Radiol Ultrasound, Malden, v.35, n.3, p.190-194, 1994.

LEMOS, R.S. Dopplerfluxometria em portadores de esquistossomose hepatoesplênica: aspectos técnicos relacionados ao cálculo do fluxo sangüíneo portal e índice de congestão. 2002. 69f. Dissertação (Mestrado, área de Cirurgia) - Universidade Federal de Pernambuco, Recife.

MACHADO, M.M. et al. Estudo Doppler na hipertensão portal. Radiol Bras, São Paulo, v.37, n.1, p.1-10, 2004.

MAMPRIM, M. J. Fígado e vesícula biliar. In: CARVALHO, C.F. (Eds.) Ultra-sonografia em pequenos animais. São Paulo: Roca, 2004. p. 51-73.

MORIYASU, F. et al. Quantitative measurement of portal blood flow in patients with cronic liver disease using an ultrasonic dupplex system consisting of a pulsed Doppler flowmeter and B-mode electroscanner. Gastroenterol, Tokyo, v.19, p.529556, 1984.

MORIYASU, F. et al. "Congestion index" of the portal vein. AJR, Philadelphia, v.46, p.735-739, 1986.

NICKEL, R. et al. The viscera of the domestic mammals. 2ed. Berlin: Verlag Paul Parey, 1979. p.114-119.

NYLAND, T.G.; FISHER, P.E. Evaluation of experimentally induced canine hepatic cirrhosis using duplex Doppler ultrasound. Vet Radiol, Malden, v.31, n.4, p.189-94, 1990.

NYLAND, T.G.; MATTOON, J.S. Ultra-som diagnóstico em pequenos animais. 2.ed. São Paulo: Roca, 2005. 469p.

O’ DONOHUE, J. et al. Diagnostic value of Doppler assessment of the hepatic and portal vessels and ultrasound of the spleen in liver disease. Eur J Gastroenterol Hepatol, London, v.16, p.147-155, 2004.

PISCAGLIA, F. et al. Influence of the spleen on portal haemodynamics: a non-invasive study with Doppler ultrasound in chronic liver disease and haematological disorders. J Gastroenterol, Tokyo, v.10, p.1220-1227, 2002. 
POWIS, R.L. AAPM Tutorial-Color flow imaging. Radiographics, Chicago, v.14, p.415-428, 1994.

ROSE, S.C.; NELSON, T.R. Ultrasonographic modalities to assess vascular anatomy and disease. JVIR, Chicago, v.15, p.25-38, 2004.

SISSON, S. Sistema digestório. In: GETTY, R. (Eds.). Anatomia dos animais domésticos. 5.ed. Rio de Janeiro: Guanabara, 1986. V.1, p.100-107.

SUGIMOTO, H. et al. Simultaneous Doppler measurement of portalvenous peak velocity, hepatic arterial peak velocity, and splenic arterial pulsatility inex for assessment of hepatic circulation. Hepato-Gastroentero, Athens, v.49, p.793-797, 2002.
SZATMARI, V. et al. Normal duplex doppler waveforms of major abdominal blood vessels in dogs: a review. Vet Radiol Ultrasound, Malden, v.42, n.2, p.93-107, 2001.

WACHSBERG, R.H. et al. Hepatofugal flow in the portal venous system: pathophysiology, imaging findings, and diagnostic pitfalls. Radiographics, Chicago, v.22, p.123-140, 2002.

WEBSTER, C.R.L. Liver and pancreatic diseases. In: ETTINGER, S.; FELDMAN E.C. (Eds.). Textbook of veterinary internal medicine. 6.ed. St. Louis: Saunders, 2005. V.2, p.1453-1464.

XIN, J.; CARLISLE, C.H. Ultrasonoraphic examination of the canine liver based on recognition of hepatic and portal veins. Vet Radiol Ultrasound, Malden, v.36, n.3, p.234-239, 1995 